

\title{
Alain Ricard, africaniste in-discipliné
}

\author{
Maëline Le Lay, Sophie Moulard
}

\section{To cite this version:}

Maëline Le Lay, Sophie Moulard. Alain Ricard, africaniste in-discipliné. Politique africaine, 2016, 143, pp.209-212. 10.3917/polaf.143.0209 . halshs-02426844

\section{HAL Id: halshs-02426844 https://shs.hal.science/halshs-02426844}

Submitted on 2 Jan 2020

HAL is a multi-disciplinary open access archive for the deposit and dissemination of scientific research documents, whether they are published or not. The documents may come from teaching and research institutions in France or abroad, or from public or private research centers.
L'archive ouverte pluridisciplinaire HAL, est destinée au dépôt et à la diffusion de documents scientifiques de niveau recherche, publiés ou non, émanant des établissements d'enseignement et de recherche français ou étrangers, des laboratoires publics ou privés. 


\section{HOMMAGE}

MAËLINE LE LAY

SOPHIE MOULARD

\section{ALAIN RICARD, AFRICANISTE IN-DISCIPLINÉ}

Alain Ricard, disparu le 27 août 2016, est l'une des figures majeures de la recherche mondiale sur les littératures de l'Afrique, un champ qu'il a participé à façonner et à reconfigurer, en fonction d'orientations qu'il a longtemps été le seul à porter en France: l'anthropologie des textes et la centralité des langues africaines. Le caractère novateur de sa recherche, comme sa capacité à susciter l'émulation autour de ses objets et de son approche singulière de la littérature et des arts d'Afrique, lui ont valu une reconnaissance internationale.

Chercheur infatigable quelques mois encore avant son décès, il était extrêmement prolixe et sur des sujets aussi variés que les langues et les politiques linguistiques (Le kiswahili, une langue moderne, 2009; Le sable de Babel. Traduction et apartheid, 2011), les récits de voyages et écrits missionnaires (édition d'Excursion missionnaire dans les Montagnes bleues de Thomas Arbousset incluant Notice sur les Zulus, 2000; Voyages de découvertes en Afrique, 2000), ou encore le théâtre (Théâtre et nationalisme. Wole Soyinka et LeRoi Jones, 1972; Mister Tameklor, suivi de Francis-le-Parisien, par le Happy Star Concert Band de Lomé, avec N. Akam, 1981; L'invention du théâtre. Le théâtre et les comédiens en Afrique noire, 1986; West African Popular Theater avec Karin Barber et John Collins, 1997; Ebrahim Hussein, théâtre swahili et nationalisme tanzanien, 1998; Wole Soyinka et Nestor Zinsou: de la scène à l'espace public, 2015).

Rédacteur en chef de Politique africaine puis directeur de la publication (1992-1995), il militait âprement pour le décloisonnement disciplinaire au nom de la centralité d'un terrain commun et de questionnements partagés par les politistes et les spécialistes des littératures et des arts, par-delà leurs objets respectifs. Arrimer fermement sa réflexion à un socle méthodologique élaboré par les anthropologues, porter une attention soutenue aux dynamiques linguistiques en œuvre dans les textes et les discours des sociétés africaines, s'intéresser aux enjeux de la «monstration ${ }^{1}$ » sur la scène artistique; autant d'angles d'approche du politique qu'il n'a eu de cesse d'explorer et de mettre en lumière au fil de ses nombreuses publications.

Dans notre dernière correspondance, quelques semaines avant son décès, Alain Ricard revenait sur certains points du très beau texte qu'il avait écrit pour la quatrième édition des Rencontre des études africaines en France (REAF), «Vertus de

1. Alain Badiou (dont les écrits sur le théâtre inspiraient beaucoup Alain Ricard) définit le théâtre comme le lieu de «monstration de la confusion»: A. Badiou (avec N. Truong), Éloge du théâtre, Paris, Flammarion, «coll. Café Voltaire», 2013, p. 70. 
Chronique de l'in-discipline

l'in-discipline: langues, textes, traductions ${ }^{2} »$. Dans ce texte qui résonne aujourd'hui comme une sorte de testament intellectuel, il est question, au fond, de l'africanisme qu'il définit comme une «in-discipline ${ }^{3} »$.

Cet africanisme qui ne va pas de soi aujourd'hui, Alain Ricard, non seulement l'assumait, mais le théorisait, d'un texte à l'autre, avec l'exigence intellectuelle qui était la sienne. Il s'attelait, dans ces deux textes à identifier les personnes et les lieux qui avaient à ses yeux porté cet africanisme dont il se revendiquait, comme à cartographier les lieux de déploiement d'une telle «méthode».

Politique africaine en faisait partie. Citant le parti-pris méthodologique du linguiste Larry Hyman dans son article «Fieldwork as a State of Mind"», il en soulignait la parenté avec l'orientation de la recherche portant sur l'Afrique telle que portée par la revue: «Rappelez-vous que j'ai longtemps été directeur de Polaf et que j'ai beaucoup travaillé avec des gens qui pensaient eux que l'Afrique était l'objet de leur intérêt scientifique, qui ne voyaient pas ça comme un faute de mieux ${ }^{5}$ ».

Le fil directeur entre toutes ces approches est donc invariablement le terrain. C'est notamment par le théâtre qu'Alain Ricard s'est frotté à l'anthropologie, et donc au terrain; autant une anthropologie du politique, à laquelle des «effrontés » comme Georges Balandier ou Jean Rouch ont redonné une vigueur nouvelle, qu'une anthropologie de la performance, et même une anthropologie des textes, dans la lignée de sa collègue et amie Karin Barber.

C'est l'anthropologie et l'expérience du terrain qui lui ont permis de recentrer les pratiques performatives et langagières au cœur de la vie sociale qui les a vues émerger. Car Alain Ricard ne s'y est pas trompé: réfutant la vision essentialiste du théâtre en Afrique telle qu'elle dominait à l'époque, il s'est rapidement arrimé aux régimes d'historicité des sociétés africaines dont il observait les créations performatives, il s'est attaché à définir leur ancrage dans les dynamiques sociales en œuvre mais aussi dans le jeu du politique, des rapports de domination.

Quotidienneté de la performance, et théâtralité de la vie quotidienne, liminarité6, immédiation ${ }^{7}$ et distanciation, autant de questionnements qui n'ont cessé d'habiter Alain Ricard, nous livrant des éclairages toujours renouvelés. Toute son œuvre confirme d'ailleurs cette mise en relation de la littérature avec les sciences humaines,

2. A. Ricard, «Vertus de l'in-discipline: langues, textes, traductions», ouverture des 4e REAF, LAM, 5 juillet 2016, <wWw.lam.sciencespobordeaux.fr/sites/lam/files/ricard_vertus_reaf_juillet_2016_0. pdf $>$, consulté le 10 octobre 2016 .

3. Cette réflexion peut se lire comme le prolongement d'un texte publié antérieurement et dans lequel il plaidait déjà pour la revendication assumée d'un positionnement africaniste. Voir A. Ricard, «Nécessaire retour de l'africanisme?», Études littéraires africaines, n 40, 2015, p. 159-175. Voir aussi A. Ricard, «De l'africanisme aux études africaines. Textes et "humanités" », Afrique E histoire, vol. 2, 2004, p. 171-192.

4. L. M. Hyman, «Fieldwork as a State of Mind», in P. Newman et M. Ratliff (dir.), Linguistic Fieldwork, Cambridge, Cambridge University Press, 2001, p. 15-31.

5. Correspondance avec Maëline Le Lay, juillet 2016.

6. V. Turner, Le phénomène rituel. Structure et contre-structure, Paris, PUF, 1990 (Traduit de The Ritual Process: Structure and Anti-Structure, Chicago, Aldine Pub. Co, 1969).

7. N. Argenti, The Intestines of State, Chicago, University of Chicago Press, 2007. 
de l'espace de la scène avec l'espace public ${ }^{8}$ : Wole Soyinka, «le poète citoyen ${ }^{9}$ » se fait le porte-voix de ce que Nestor Zinsou subissait au Togo à l'époque d'Eyadema père ${ }^{10}$, d'une façon comparable à ce que les rappeurs entreprendront de faire plus tard dans le contexte des alternances démocratiques ${ }^{11}$. Du concert party au rap, Alain Ricard a toujours su saisir l'enjeu qu'il y avait à s'intéresser aux formes de ruse et d'insoumission que chaque époque successive a vu naître ${ }^{12}$. «Cette anthropologie du théâtre cherche à retracer un parcours de parole et du texte dans une région de l'Afrique » dit-il, avant d'ajouter: «Dans mon cas précis, elle sert de soubassement à une lecture des œuvres dramatiques et veut tout simplement apporter une réponse simple à une question récurrente: [...] Pourquoi ai-je l'impression de mieux comprendre une partie de la condition africaine après les avoir lus ?13" .

Le théâtre et les textes l'ont conduit vers l'anthropologie, comme l'anthropologie l'a conduit à s'intéresser au politique en Afrique, toujours par le truchement de l'in-discipline. Formant une boucle de sens, le politique en Afrique, éclairé par les sciences humaines, est ce qui le ramène finalement vers les textes, et vers leur lecture incarnée, contextualisée ${ }^{14}$. Ses livres plus généralistes, mais toujours novateurs, sont caractérisés par la volonté de briser la dichotomie oral/écrit et de montrer qu'en fait, les deux dimensions se nourrissent l'une l'autre. Ils sont aujourd'hui des ouvrages de référence pour tous les étudiant(e)s, chercheur(e)s et curieux des littératures de l'Afrique: Littératures d'Afrique noire: des langues aux livres (1995 et 2004 pour la traduction anglaise) et Histoire des littératures de l'Afrique subsaharienne (2006) 15 .

C'est dans ce même esprit que l'on peut aussi lire son travail de cinéaste. Son premier film, tourné dans les années 1980 et numérisé en 2011, Le principe d'Asihu, porte sur le concert-party au Togo. Il est l'auteur de deux autres films tournés au Togo et a également participé, en tant que conseiller littéraire, au film de Bankolé Bello, Wole Soyinka, poète citoyen, où il apparaît en conversation avec l'écrivain, Prix Nobel

8. À propos de Soyinka et Zinsou: «Actes et écrits fonctionnent ensemble; c'est ainsi qu'ils conçoivent le théâtre, [...] que le discours théâtral puisse soudain éclater sur la place publique. Leurs écrits sont pénétrés de cet écho imaginé. La scène théâtrale est une scène publique pour eux»: A. Ricard, Wole Soyinka et Nestor Zinsou: De la scène à l'espace public. Politique et religion, Paris, Karthala, coll. «Lettres du Sud», 2015, p. 15. Au sujet de cet ouvrage, voir la recension de M. Le Lay dans Politique africaine, $\mathrm{n}^{\circ}$ 39, 2015, p. 204-205.

9. Voir le film de Bankole Bello, Wole Soyinka, poète citoyen, ARTE/FR3/La Sept, Nigeria, 1993, $52 \mathrm{~min}$. 10. «Ce que Zinsou vivait, Soyinka le dénonçait dans ses pièces satiriques». Voir A. Ricard, Wole Soyinka et Nestor Zinsou..., op. cit., p. 12.

11. Au Sénégal et au Burkina Faso notamment.

12. Voir A. Ricard, «Les Parisiens du concert. Discours métissé ou discours dominé? », Politique africaine, $\mathrm{n}^{\circ}$ 5, 1982, p. 35-46. Et, en hommage à l'œuvre d'Alain Ricard, S. Moulard, «Quand se représenter veut dire exister: du concert party au rap, quarante ans de pratiques performatives en Afrique subsaharienne», in V. Coulon et X. Garnier (dir.), Les littératures africaines; Textes et terrains, Paris, Karthala, coll. «Lettres du Sud», 2011, p. 15-30.

13. A. Ricard, Wole Soyinka, et Nestor Zinsou, op. cit., p. 18.

14. William Labov, dont Alain Ricard était un lecteur assidu, avait mis en lumière les rapports de force qui s'exercent à travers le choix de langues des locuteurs.

15. Pour consulter la liste exhaustive des ouvrages d'Alain Ricard, voir sa page sur le site du LAM (Les Afriques dans le monde), <lam.sciencespobordeaux.fr/users/alain-ricard>, consulté le 10 octobre 2016. 
Chronique de l'in-discipline

de littérature en 1986. Ses deux films les plus récents ont été tournés à Bayreuth en Allemagne où il avait noué de fortes et durables relations dans le milieu africaniste: Aquitaine, Afriques: Contact, Zones: une visite de l'exposition d'Iwalewahaus (2012) et Nestor Zinsou à Bayreuth (2013).

L'expérience du terrain est encore celle qui a conduit Alain Ricard à s'intéresser aux langues des textes qu'il étudiait, textes poétiques, dramatiques ou narratifs. $\mathrm{Ou}$, plus exactement, c'est en circulant dans les villes africaines, au gré de sa collecte de livres et de ses rencontres avec les artistes, que les langues se sont adressées à lui. En dressant de lui-même un portrait de «chiffonnier» et de «linguiste de terrain », il écrivait: «le monde nous saute à la figure, à nous de nous en débrouiller ${ }^{16}$ ». Acceptant dès lors la confrontation du chercheur avec «le mur de perplexité» qu'impliquent non seulement l'exposition intensive à une langue inconnue, mais surtout, l'économie linguistique complexe de pays plurilingues, Alain Ricard a toujours porté une attention soutenue à la place des langues et aux fonctions sociales de leurs usages dans les sociétés où il se trouvait.

C'est cette même volonté politique qu'il saluait dans son essai, Le sable de Babel. Traduction et apartheid ${ }^{17}$. Il y montrait combien la politique linguistique de l'Afrique du Sud post-apartheid qui choisit d'adopter 11 langues officielles, en faisant de la traduction un fonctionnement élémentaire et indispensable de la communication, dessinait symboliquement le socle d'une nouvelle communauté humaine dont l'avènement ne pouvait qu'être accueilli avec soulagement après le paradigme de l'exclusion et de négation de l'altérité qui avait caractérisé la période de l'apartheid.

L'attention qu'il n'a cessé de porter à la gestion des langues en présence dans une société donnée - et plus précisément, son analyse de ce processus dans un discours ou un texte - remonte aux premiers pas de son parcours en Afrique, au Togo. Le concert party lui offrit un observatoire de choix pour observer la gestion, par les comédiens, des langues ewe et française selon les différentes situations d'interaction et selon les publics. Comme il le montre dans son film, Le principe d'Asihu ${ }^{18}$ et dans son petit essai, L'invention du théâtre - ouvrage majeur sur le théâtre en Afrique ${ }^{19}$ - sur le plateau de jeu, s'incarnait la dimension éminemment politique de l'usage des langues.

Ce parcours de recherche extrêmement riche témoigne bien de ce que représentaient le travail et la personnalité d'Alain Ricard dans le domaine des humanités: il était avant tout un passeur de frontières qui, inlassablement, s'employait, non sans provocation d'ailleurs, à briser les enclos disciplinaires en vue de fédérer l'ensemble des sciences humaines autour d'un objet: 1'Afrique.

Maëline Le Lay et Sophie Moulard Les Afriques dans le monde (LAM), Sciences Po Bordeaux

16. A. Ricard, «Vertus de l'in-discipline... », art. cité.

17. A. Ricard, Le sable de Babel. Traduction et apartheid, Paris, CNRS éditions, 2011.

18. Le Principe d'Asihu, tourné dans années 1980 à Lomé et récemment réédité, est un film documentaire présentant le travail des Happy Star, un des groupes mythiques de concert-party du Togo (A. Ricard, Le Principe d'Asihu, CNRS Images, 2011).

19. A. Ricard, L'Invention du théâtre: le théâtre et les comédiens en Afrique noire, Lausanne, L'Âge d'Homme, 1986. 